\title{
Call on the reserve: Coronary vasomotor dysfunction is a potential biomarker of cardiovascular risk in patients with breast cancer
}

\author{
Michael T. Osborne, MD, ${ }^{a}$ Simran Grewal, DO, ${ }^{a}$ and Tomas G. Neilan, $M D^{a}$ \\ a Cardiology Division, Cardiovascular Imaging Research Center, Massachusetts General Hospital \\ and Harvard Medical School, Boston, MA
}

Received Sep 29, 2021; accepted Sep 29, 2021

doi: $10.1007 / \mathrm{s} 12350-021-02831-3$

See related article, pp. 3072-3081

Coronary vasomotor dysfunction in the absence of obstructive epicardial coronary artery disease (CAD) associates with adverse cardiovascular events in an array of cardiac and non-cardiac diseases, including hypertrophic cardiomyopathy, obesity, and chronic kidney disease. $^{1-3}$ Myocardial perfusion imaging (MPI) with positron emission tomography (PET) facilitates the assessment of coronary vasomotor dysfunction through quantification of myocardial flow reserve (MFR) as the ratio of peak stress to rest myocardial blood flow. ${ }^{4}$ In individuals without obstructive CAD, reduced MFR is a marker of subclinical cardiovascular disease (CVD) that may be indicative of impaired vasomotor function, myocardial fibrosis or inflammation, or diffuse atherosclerosis. $^{5}$

CVD is a leading cause of morbidity and mortality in patients with prior or current breast cancer. This association has been linked in part to overlapping risk factors between the two diseases (e.g., obesity, tobacco use, and lack of exercise), shared biology, and the

Funding Dr. Osborne is supported by the United States National Institutes of Health/National Heart, Lung, and Blood Institute (NIH/ NHLBI) Grant \#K23HL151909. Dr. Neilan is supported by a gift from A. Curt Greer and Pamela Kohlberg and the NIH/NHLBI Grants \#R01HL130539, \#R01HL137562, and \#K24HL150238.

Reprint requests: Michael T. Osborne, MD, Cardiology Division, Cardiovascular Imaging Research Center, Massachusetts General Hospital and Harvard Medical School, 55 Fruit St., Yawkey 5E, Boston, MA 02114-2750; mosborne@partners.org

J Nucl Cardiol 2022;29:3082-5.

$1071-3581 / \$ 34.00$

Copyright (c) 2021 American Society of Nuclear Cardiology. adverse cardiovascular effects of standard cancer treatments such as cytotoxic chemotherapies (e.g., anthracyclines), targeted therapies (e.g., trastuzumab), immune therapies, and thoracic radiation that are each linked in some part to heart failure, accelerated atherosclerosis, and cardiovascular events. ${ }^{6}$ Women treated for breast cancer also experience a marked impairment in functional capacity. Specifically, women treated for breast cancer have a 20-30\% lower functional capacity, $77 \%$ of breast cancer survivors have a peak oxygen consumption (measured as peak $\mathrm{VO}_{2}$ ) that is below the 20th percentile for their age group, and the peak $\mathrm{VO}_{2}$ of a 40-year-old woman treated for breast cancer with chemotherapy and radiation therapy is similar to that of a 70-year-old healthy, sedentary woman. ${ }^{7,8}$ In breast cancer patients with higher CVD risk receiving anthracyclines, current guidelines recommend echocardiography at baseline, upon therapy completion, and six months after therapy completion. ${ }^{6,9,10}$ Those receiving trastuzumab are additionally recommended to undergo echocardiography every three months during therapy. ${ }^{6,9}$ It is also recommended that patients receiving these therapies routinely undergo assessment of electrocardiograms, troponin, and B-type natriuretic peptide. ${ }^{6,9,11}$ This surveillance facilitates identification of patients with abnormalities suggestive of subclinical cardiac dysfunction due to cardiotoxic therapies principally to consider initiation of cardioprotective medical therapy (e.g., beta-blockers, angiotensin receptor blockers, and angiotensin-converting enzyme inhibitors). ${ }^{6,9,12}$ Similarly, guidelines recommend that breast cancer patients receiving radiation therapy should undergo echocardiography at baseline with follow-up echocardiography and stress testing five to ten years later depending on their baseline CVD risk. ${ }^{13}$ Guidelines currently consider nuclear and 
magnetic resonance imaging to be alternative methods for the evaluation and monitoring of patients receiving cardiotoxic therapies. ${ }^{9}$ Notably, additional routine cardiovascular surveillance is not currently recommended for breast cancer patients who do not receive cardiotoxic therapies.

The assessment of MFR as a marker of coronary vasomotor dysfunction with PET MPI in patients with breast cancer may provide a novel means of evaluating for subclinical CVD and cardiovascular risk in this population. In addition to the adverse effects on MFR of shared cardiovascular and breast cancer risk factors, radiation and doxorubicin have also both been associated with impaired vasomotor function. ${ }^{1,14-17}$ In a recent study, Groarke et al showed for each Gray increase in mean cardiac radiation dose, there was a graded decrease in global MFR as well as MFR in the left anterior descending coronary artery distribution. ${ }^{15}$ Accordingly, by accounting for the adverse effects of both pre-existing risk factors and malignancy therapies, MFR could provide a synergistic assessment of cardiovascular risk in this population. Nevertheless, prior to the current study, the relationship between coronary vasomotor dysfunction and adverse events had not previously been studied in a general cohort of individuals with known malignancy.

In this issue of the Journal of Nuclear Cardiology, Divakaran et al seek to address this knowledge gap by testing the hypothesis that abnormal MFR could serve as a predictor of cardiovascular risk in a retrospective population of breast cancer patients. ${ }^{18}$ They evaluated a cohort of 87 consecutive patients with prior or active breast cancer and no clinically overt CAD, left ventricular dysfunction (left ventricular ejection fraction $\geq 45 \%$ ), or abnormal myocardial perfusion who underwent clinically indicated vasodilator PET MPI with MFR quantification between 2006 and 2017 at a single center to report on the relationship between MFR and adverse cardiovascular events. The study sample was almost entirely female $(98.9 \%)$, and many had received chemotherapy $(31.0 \%)$ and thoracic radiation $(65.5 \%)$. There was a median 7.9 years between breast cancer diagnosis and PET MPI. Over a median follow-up interval of 7.6 years after imaging, 15 patients experienced major adverse cardiovascular events (MACE, defined as cardiovascular death, non-fatal myocardial infarction, heart failure admission, or coronary revascularization), and there were 23 total deaths. MFR as both a continuous variable and as tertiles associated with MACE in models adjusted for the competing risk of allcause death as well as the Morise scale, a validated assessment of pre-test probability that incorporates standard cardiovascular risk factors, and chronic kidney disease. $^{19}$ Although a non-significant trend was observed, there was no association between MFR and all-cause death. Of note, the lowest tertile of MFR included individuals with markedly reduced global MFR of $<1.71$.

Several interesting observations were made within these primary findings. Impaired MFR had no association with coronary artery calcification, which is similar to findings in a separate small pilot study of breast cancer patients who received radiation. ${ }^{20}$ Further, among those with in the lowest tertile of MFR there was a pattern of high resting flow with relatively reduced peak stress flow. These findings collectively suggest a mechanism of coronary vasomotor dysfunction in this population in which impaired flow augmentation appears more common than diffuse atherosclerosis. There was also no association between MFR and malignancy treatment (i.e., chemotherapy, surgery, and thoracic radiation) in this cohort. The authors conclude that MFR may serve as marker of cardiovascular fitness and facilitate improved cardiovascular risk stratification among patients with prior or current breast cancer (Figure 1).

The study should be interpreted within the context of its design. This was a retrospective single center study with a population that was referred primarily for the assessment of cardiovascular symptoms, and the sample size was modest with relatively few adverse events. Accordingly, it was unable to evaluate whether MFR measurements were more predictive of adverse events in different subpopulations of breast cancer patients (e.g., those that received chemotherapy or radiation). Further, the study design did not provide MFR results before and after different treatments to determine the effect of preexisting risk factors and cancer therapies on coronary vasomotor dysfunction.

These findings support the need for further studies to better understand the prognostic implications of coronary vasomotor dysfunction in a broad population of patients with breast cancer and not only those with symptoms or a clinical indication for PET MPI. Further, the clinical implications of impaired MBF will need to be compared with those of other more widely implemented measures such as ejection fraction and strain derived from echocardiography and serological biomarkers. Additionally, the impacts of chemotherapy and radiation and their interaction with traditional risk factors on coronary vasomotor dysfunction could be evaluated with PET MPI before and after cancer therapy, especially as the field of breast cancer treatment is changing with increasing approvals for immune-based therapies. Through such a study, the population of individuals who would benefit most from PET MPI, a relatively costly and limited resource, could be identified. In subsequent studies, the impact of therapies on 


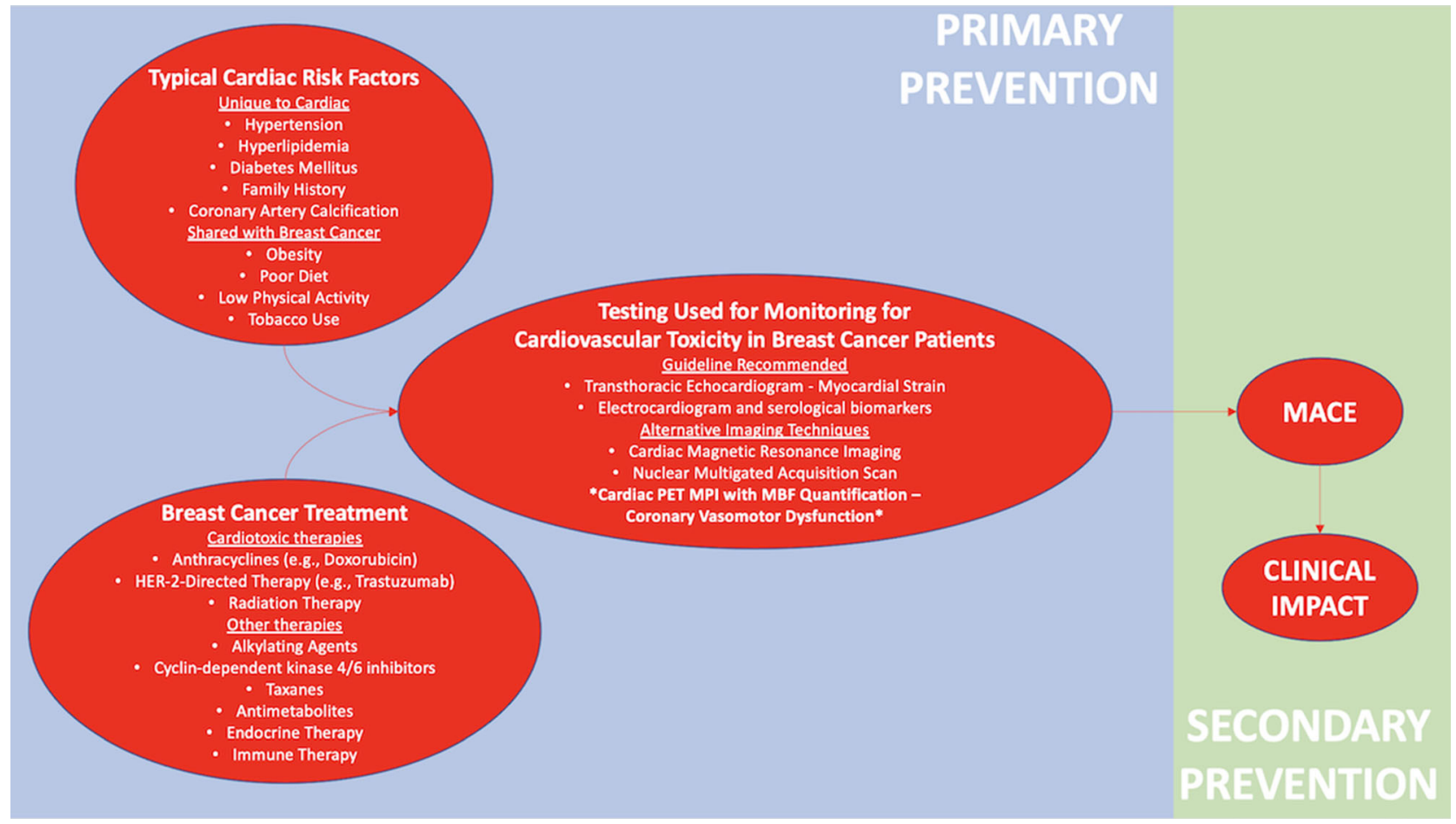

Figure 1. Monitoring for cardiovascular disease resulting from the convergence of baseline risk factors and the effects of cancer therapies in breast cancer patients.

MFR and CVD events could also be assessed. Finally, the finding that abnormal MFR does not associate with coronary artery calcification and largely stems from a failure to appropriately augment high resting myocardial blood flow suggests that the underlying mechanism of coronary vasomotor dysfunction in breast cancer merits further investigation.

Among patients with a history of breast cancer, coronary microvascular dysfunction detected by PET MPI appears to serve as an effective biomarker for cardiovascular risk. Further work is needed to determine how MFR performs compared to guideline recommended testing and to identify those who would benefit most from its measurement among patients with breast cancer. Nevertheless, the time has come that MFR should no longer be kept in reserve for the assessment of cardiovascular risk in malignancy patients.

\section{Disclosures}

Dr. Osborne receives consulting fees from WCG Intrinsic Imaging for unrelated work. Dr. Neilan has received grant funding from AstraZeneca and advisory fees from Parexel, AbbVie, H3 Biomedicine, Genentech, Aprea Therapeutics, $B M S$, and WCG Intrinsic Imaging for unrelated work.

\section{References}

1. Bajaj NS, Osborne MT, Gupta A, Tavakkoli A, Bravo PE, Vita T. Coronary microvascular dysfunction and cardiovascular risk in obese patients. J Am Coll Cardiol 2018;72:707-17.

2. Bajaj NS, Singh A, Zhou W, Gupta A, Fujikura K, Byrne C, et al. Coronary microvascular dysfunction, left ventricular remodeling, and clinical outcomes in patients with chronic kidney impairment. Circulation 2020;141:21-33.

3. Cecchi F, Olivotto I, Gistri R, Lorenzoni R, Chiriatti G, Camici PG. Coronary microvascular dysfunction and prognosis in hypertrophic cardiomyopathy. N Engl J Med 2003;349:1027-35.

4. Murthy VL, Bateman TM, Beanlands RS, Berman DS, BorgesNeto S, Chareonthaitawee $P$, et al. Clinical quantification of myocardial blood flow using PET: Joint position paper of the snmmi cardiovascular council and the ASNC. J Nucl Cardiol 2018;25:269-97.

5. Taqueti VR, Di Carli MF. Coronary microvascular disease pathogenic mechanisms and therapeutic options: JACC state-ofthe-art review. J Am Coll Cardiol 2018;72:2625-41.

6. Mehta LS, Watson KE, Barac A, Beckie TM, Bittner V, CruzFlores S, et al. Cardiovascular disease and breast cancer: Where these entities intersect: A scientific statement from the American Heart Association. Circulation 2018;137:e30-66.

7. Jones LW, Courneya KS, Mackey JR, Muss HB, Pituskin EN, Scott JM, et al. Cardiopulmonary function and age-related decline across the breast cancer survivorship continuum. J Clin Oncol 2012;30:2530-7.

8. Peel AB, Thomas SM, Dittus K, Jones LW, Lakoski SG. Cardiorespiratory fitness in breast cancer patients: A call for normative values. J Am Heart Assoc 2014;3:e000432. 
9. Plana JC, Galderisi M, Barac A, Ewer MS, Ky B, Scherrer-Crosbie M, et al. Expert consensus for multimodality imaging evaluation of adult patients during and after cancer therapy: A report from the American Society of Echocardiography and the European Association of Cardiovascular Imaging. Eur Heart J Cardiovasc Imaging 2014;15:1063-93.

10. Armenian SH, Lacchetti C, Lenihan D. Prevention and monitoring of cardiac dysfunction in survivors of adult cancers: American society of clinical oncology clinical practice guideline summary. J Oncol Pract 2017;13:270-5.

11. Yancy CW, Jessup M, Bozkurt B, Butler J, Casey DE Jr, Colvin MM, et al. 2017 ACC/AHA/HFSA focused update of the 2013 ACCF/AHA guideline for the management of heart failure: A report of the American College of Cardiology/American Heart Association Task Force on Clinical Practice Guidelines and the Heart Failure Society of America. Circulation 2017;136:e137-61.

12. Cardinale D, Colombo A, Lamantia G, Colombo N, Civelli M, De Giacomi G, et al. Anthracycline-induced cardiomyopathy: Clinical relevance and response to pharmacologic therapy. J Am Coll Cardiol 2010;55:213-20.

13. Lancellotti P, Nkomo VT, Badano LP, Bergler-Klein J, Bogaert J, Davin L, et al. Expert consensus for multi-modality imaging evaluation of cardiovascular complications of radiotherapy in adults: A report from the European Association of Cardiovascular Imaging and the American Society of Echocardiography. Eur Heart J Cardiovasc Imaging 2013;14:721-40.

14. Banister HR, Hammond ST, Parr SK, Sutterfield SL, Turpin VG, Treinen S, et al. Lower endothelium-dependent microvascular function in adult breast cancer patients receiving radiation therapy. Cardiooncology 2021;7:18.

15. Groarke JD, Divakaran S, Nohria A, Killoran JH, Dorbala S, Dunne RM, et al. Coronary vasomotor dysfunction in cancer survivors treated with thoracic irradiation. J Nucl Cardiol 2020. h ttps://doi.org/10.1007/s12350-020-02255-5.

16. Hader SN, Zinkevich N, Norwood Toro LE, Kriegel AJ, Kong A, Freed JK, et al. Detrimental effects of chemotherapy on human coronary microvascular function. Am J Physiol Heart Circ Physiol 2019;317:H705-10.

17. Rooks C, Faber T, Votaw J, Veledar E, Goldberg J, Raggi P, et al. Effects of smoking on coronary microcirculatory function: A twin study. Atherosclerosis 2011;215:500-6.

18. Divakaran S, Caron JP, Zhou W, Hainer J, Bibbo CF, Skali H et al. Coronary Vasomotor Dysfunction Portends Worse Outcomes in Patients with Breast Cancer. J Nucl Cardiol 2021.

19. Morise AP, Haddad WJ, Beckner D. Development and validation of a clinical score to estimate the probability of coronary artery disease in men and women presenting with suspected coronary disease. Am J Med 1997;102:350-6.

20. Nehmeh SA, Fox JJ, Schwartz J, Ballangrud AM, Schoder H, Zhao $\mathrm{Y}$, et al. A pilot study of (13) N-ammonia cardiac PET imaging to assess subacute cardiotoxicity following adjuvant intensity-modulated radiotherapy for locally advanced breast cancer. Clin Imaging 2020;68:283-90.

Publisher's Note Springer Nature remains neutral with regard to jurisdictional claims in published maps and institutional affiliations. 